\title{
Efficacy and Safety of Massage for Osteoarthritis of the Knee: a Randomized Clinical Trial
}

\author{
Adam Perlman, MD, MPH' , Susan Gould Fogerite, PhD², Oliver Glass, PhD ${ }^{7}$, Elizabeth Bechard, BA? , \\ Ather Ali, ND, MPH, MHS ${ }^{3}$, Valentine Y. Njike, MD, MPH ${ }^{4}$, Carl Pieper, DPH ${ }^{7}$, Natalia O. Dmitrieva, PhD ${ }^{5}$, \\ Alison Luciano, MPH, PhD ${ }^{7}$, Lisa Rosenberger, ND, LAc ${ }^{4}$, Teresa Keever, MSHS, BSN, RN ${ }^{7}$, \\ Carl Milak, $A A^{2}$, Eric A. Finkelstein, $P h D, M H A^{6}$, Gwendolyn Mahon, MSc, PhD ${ }^{2}$, \\ Giovanni Campanile, $M D^{7}$, Ann Cotter, $M D^{8}$, and David L. Katz, MD, $\mathrm{MPH}^{4}$
}

'Duke Integrative Medicine, Durham, NC, USA; ${ }^{2}$ Rutgers School of Health Professions, Institute for Complementary and Alternative Medicine, Newark, NJ, USA; ${ }^{3}$ Yale School of Medicine, New Haven, CT, USA; ${ }^{4}$ Yale-Griffin Prevention Research Center, Griffin Hospital, Yale University School of Public Health, Derby, CT, USA; ${ }^{5}$ Department of Psychological Sciences, Northern Arizona University, Flagstaff, AZ, USA; ${ }^{6}$ Duke-NUS Medical School, Singapore, Singapore; ${ }^{7}$ Atlantic Integrative Medical Associates, Chambers Center for Well Being, Morristown, NJ, USA; ${ }^{8}$ Veterans Administration New Jersey Health Care Center, East Orange, NJ, USA.

BACKGROUND: Current treatment options for knee osteoarthritis have limited effectiveness and potentially adverse side effects. Massage may offer a safe and effective complement to the management of knee osteoarthritis.

OBJECTIVE: Examine effects of whole-body massage on knee osteoarthritis, compared to active control (lighttouch) and usual care.

DESIGN: Multisite RCT assessing the efficacy of massage compared to light-touch and usual care in adults with knee osteoarthritis, with assessments at baseline and weeks 8 , $16,24,36$, and 52. Subjects in massage or light-touch groups received eight weekly treatments, then were randomized to biweekly intervention or usual care to week 52 . The original usual care group continued to week 24. Analysis was performed on an intention-to-treat basis.

PARTICIPANTS: Five hundred fifty-one screened for eligibility, 222 adults with knee osteoarthritis enrolled, 200 completed 8-week assessments, and 175 completed 52week assessments.

INTERVENTION: Sixty minutes of protocolized full-body massage or light-touch.

MAIN MEASURES: Primary: Western Ontario and McMaster Universities Arthritis Index. Secondary: visual analog pain scale, PROMIS Pain Interference, knee range of motion, and timed 50-ft walk.

KEY RESULTS: At 8 weeks, massage significantly improved WOMAC Global scores compared to light-touch $(-8.16,95 \% \mathrm{CI}=-13.50$ to -2.81$)$ and usual care $(-$ $9.55,95 \% \mathrm{CI}=-14.66$ to -4.45$)$. Additionally, massage improved pain, stiffness, and physical function WOMAC

Prior Presentations Preliminary results from the study were presented at the 2016 World Congress on Osteoarthritis in Amsterdam, April 2016, and the 2016 International Congress on Integrative Medicine and Health in Las Vegas, May 2016.

Ather Ali is deceased. This paper is dedicated to his memory.

Electronic supplementary material The online version of this article (https://doi.org/10.1007/s11606-018-4763-5) contains supplementary material, which is available to authorized users.

Received March 6, 2018

Revised May 30, 2018

Accepted November 20, 2018

Published online December 12, 2018 subscale scores compared to light-touch $(p<0.001 ; p=$ $0.04 ; p=0.02$, respectively) and usual care $(p<0.001 ; p=$ $0.002 ; p=0.002$; respectively). At 52 weeks, the omnibus test of any group difference in the change in WOMAC Global from baseline to 52 weeks was not significant ( $p=$ 0.707, $\mathrm{df}=3$ ), indicating no significant difference in change across groups. Adverse events were minimal.

CONCLUSIONS: Efficacy of symptom relief and safety of weekly massage make it an attractive short-term treatment option for knee osteoarthritis. Longer-term biweekly dose maintained improvement, but did not provide additional benefit beyond usual care post 8-week treatment.

TRIAL REGISTRATION: clinicaltrials.gov NCT01537484

KEY WORDS: massage; osteoarthritis; arthritis; knee pain; musculoskeletal pain.

$\mathrm{J}$ Gen Intern Med 34(3):379-86

DOI: $10.1007 / \mathrm{s} 11606-018-4763-5$

(c) Society of General Internal Medicine 2018

\section{INTRODUCTION}

Osteoarthritis is a degenerative disease affecting approximately 30 million Americans. ${ }^{1-3}$ A leading cause of disability worldwide, ${ }^{4}$ osteoarthritis poses significant economic strain. Patients often face either surgical interventions or the burden of debilitating joint pain, weakness, and loss of function if symptoms cannot be effectively managed. $^{5-12}$ While physicians generally recommend pharmacotherapy for osteoarthritis, concerns over its limited effectiveness and associations with adverse events are growing. Collectively, these concerns have reduced public confidence in use of pharmaceuticals for osteoarthritis and increased interest in therapies perceived to be safer. ${ }^{13-15}$

Massage therapy is one of the most popular complementary medicine interventions: in 2012, 15.4 million Americans reported using massage for osteoarthritis. ${ }^{13-20}$ Despite its widespread use, only a small body of research exists exploring massage efficacy to improve osteoarthritis symptoms. In 2006, we first reported 
results of a pilot study of massage for knee osteoarthritis. ${ }^{19}$ Subjects receiving massage demonstrated significant improvements in pain, stiffness, and physical function at 8 weeks, with some positive effects persisting to 16 weeks.

We subsequently developed a formal protocol ${ }^{21}$ for the massage intervention and tested it in four 8-week "doses" of varying frequency and duration to determine an optimal, practical dose for adults with knee osteoarthritis. Our randomized controlled trial (RCT) demonstrated that a 60-min weekly dose was clinically superior compared to 30-min doses (weekly or biweekly) and similar to 60-min biweekly doses. ${ }^{22}$

Here, we report on a multisite RCT with a primary aim of assessing efficacy of 8 weeks of 60-min weekly Swedish massage compared to light-touch and usual care in 222 adults with knee osteoarthritis. Secondary aims were to assess long-term effects of 8 weeks of massage and the utility of lower-dose "maintenance" massage.

\section{METHODS}

Eligible participants were individuals with radiographically established knee osteoarthritis who met American College of Rheumatology criteria, ${ }^{23}$ were at least 35 years old, and had a baseline score of 40-90 (out of 100) for knee pain on the visual analog pain scale. Subjects using non-steroidal anti-inflammatory drugs or other analgesics were included if their doses remained stable for 3 months prior to the intervention.

People with serious medical conditions, dual knee replacement, recent use of corticosteroids or hyaluronate, knee arthroscopy or knee injury within the past year, or regular use of massage therapy were excluded.

Recruitment took place between June 2012 and May 2014 at Duke University (Durham, NC), Rutgers University (Newark, NJ), Atlantic Health System (Morristown, NJ), and Griffin Hospital (Derby, CT) through flyers, newspaper advertisements, and press releases. Informational letters were sent to patients who responded to these advertisements or were referred from rheumatologists and primary care physicians at the respective locations.

Those who passed screening, provided physician confirmation of osteoarthritis, and consented were randomized. All study materials were approved by the Institutional Review Boards of participating sites. All subjects received gift certificates for 3 future massages as compensation for their participation.

\section{Randomization}

Subjects $(n=222)$ were randomized to one of 3 study arms: massage, light-touch, or usual care, using a permuted design in a 1:1:1 ratio and stratified by site and baseline body mass index (BMI $<30$ vs. $\geq 30$ ). A second randomization was performed for the massage and light-touch groups following the 8-week assessment, where subjects were assigned in a 1:1 ratio at each site, stratified by baseline BMI, to continue with massage or light-touch (but biweekly), or no treatment (usual care) for the remainder of the study. All subjects initially randomized to usual care received weekly massage from weeks 25 to 32 and then were randomized to either biweekly massage or usual care for 16 weeks (data not included in this report). Block (6) random allocation sequences were generated by a statistician at the Yale-Griffin Prevention Research Center, given to unblinded research assistants who enrolled participants accordingly and reported allocation within $48 \mathrm{~h}$. Subject participation, randomization, and flow are shown in Figures 1 and 2.

\section{Study Design and Intervention}

The study was a multisite RCT designed to assess the initial and long-term effects of an 8-week course of weekly 60-min massages and the utility of biweekly maintenance dosing. Swedish massage was compared to weekly 60 -min light-touch treatments (active control) and usual care (passive control) at 8 weeks (primary study endpoint), 16 weeks, and 24 weeks. For subjects who received the initial 8 weeks of massage, biweekly massage was compared to no further intervention (usual care) and to lighttouch followed by biweekly maintenance or no further treatment out to 52 weeks. Usual care was defined across the sites as a subject's typical care regimen for their osteoarthritis, provided that all subject inclusion and exclusion criteria were met. The usual care group received no intervention for the first 24 weeks of the study, then was crossed over to 8 weeks of massage, then was randomized to either maintenance massage or usual care for the remainder of the study (Fig. 2).

The massage and light-touch groups were assessed at baseline and $8,16,24,36$, and 52 weeks. The original usual care group was assessed at baseline and 8,16 , and 24 weeks, then postcrossover to 8 weeks of massage, assessed at 32, 40, and 48 weeks (data post week 24, not shown). At each site, visits were scheduled by an unblinded research assistant, while study coordinators, blinded to treatment assignment, conducted assessments.

Participants randomized to massage received $60 \mathrm{~min}$ of whole-body massage, which followed the standardized protocol of our previous studies (Supplemental Table 1). ${ }^{19}, 21,22$ The light-touch protocol was developed by Patterson et al. to control for the non-active effects of massage therapy. ${ }^{24}$ This protocol involves the massage therapist gently placing their hands in a specified sequence on the major muscle groups and joints of the participant for a total of $60 \mathrm{~min}$. Massage therapists made clinical notes and signed a form after each treatment attesting to adherence to study protocols or noting deviations. The same massage therapists delivered both the massage and light-touch interventions. More information on intervention fidelity can be found in Supplemental Table 2.

Study data were collected and managed using REDCap electronic data capture tools hosted at Duke University.

Outcome Measures. Primary Outcome Measure. The primary outcome was the between-group change in the WOMAC Global score (0 to 100 scale; WOMAC 


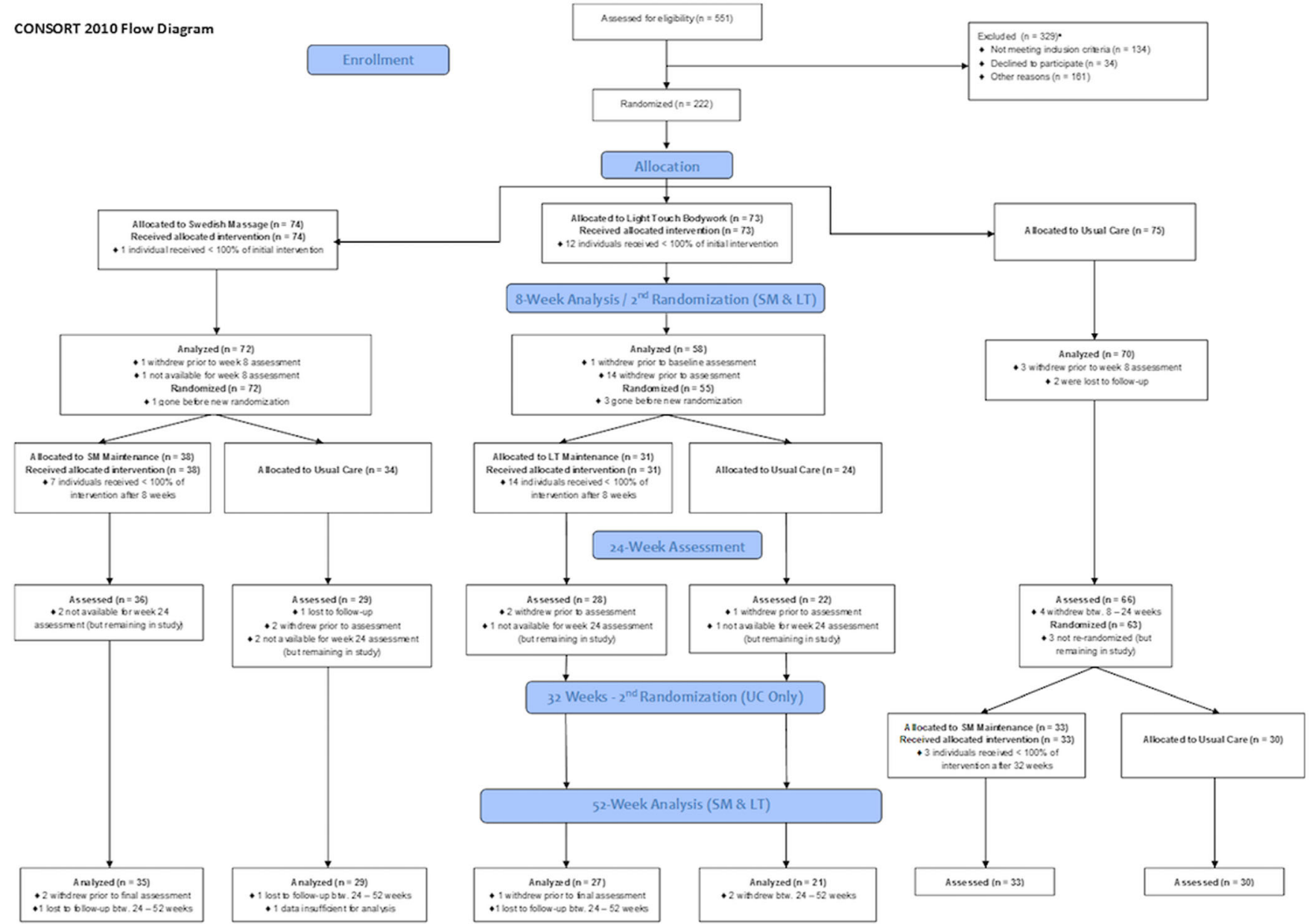

Figure 1 Participant flow diagram. a Exclusion criteria were as follows: previous diagnosis of osteoarthritis; age younger than 35 years; diagnosis of rheumatoid arthritis, fibromyalgia, and recurrent or active pseudogout; presence of cancer or other decompensating conditions; kidney or liver failure; asthma requiring the use of steroids or oral corticosteroids within past 4 weeks; use of intra-articular corticosteroids within the past 12 weeks; use of hyaluronate within the past 6 months; knee arthroscopy within the past year; knee replacement on study knee; significant knee injury within past 6 months; presence of a rash or open wound over the knee; current regular use of massage therapy.

Global). ${ }^{25-27}$ The WOMAC subscales assess dimensions of pain, functionality, and joint stiffness through 24 questions $(0$ to 100 visual analog scale). It has been subjected to numerous validation studies ${ }^{25,26}$ and was successfully utilized in the pilot $^{19}$ and dose-finding studies. ${ }^{22}$

Secondary Outcome Measures. Secondary measures included pain as measured on the visual analog pain scale (0 to 100 scale; VAS $)^{28}$ and the NIH PROMIS Pain Interference Questionnaire, a validated 6-question scale extensively tested in adult populations for reliability and comparability to other pain measures. ${ }^{29}$ Other outcomes were range of motion (ROM) allowed at the knee during flexion using a double-armed goniometer and 50-ft timed walk on a smooth level surface.

Statistical Analysis. Primary analyses assessed therapeutic effect at 8 weeks and secondarily maintenance effect at 52 weeks. Therapeutic effect analyses followed intention-totreat principles as closely as possible. Linear regression models were used to predict change in scores, adjusted for baseline, BMI
$(<30 / 30+)$, and study site. Maintenance effect and therapeutic maintenance durability analyses were performed using modified intention-to-treat methodology and we used mixed model analysis with time invariant adjustment for BMI $(<30 / 30+)$, study site, and included participants as random intercepts. Time variant main effects included nominal occasion of measurement, randomization group, and randomization-by-occasion interaction terms. Additional exploratory analyses were performed to assess the combined therapy and maintenance effect (i.e., whole treatment effect) using linear regression to predict change in scores, adjusted for baseline, BMI $(<30 / 30+)$, and study site. Values are presented as predicted means with $95 \%$ confidence intervals unless otherwise stated. All possible pairwise contrasts were reported using uniform notation (A vs. B), where reported values represent the B from A difference term. Additional analyses compared rates of adverse events (AEs) and treatment discontinuation by treatment arm using Fisher's exact tests. $p$ values and confidence intervals are unadjusted for multiple testing. All analyses were conducted in Stata/SE Version 14.0 (Stata Corp).

The sample size was estimated to provide $\geq 90 \%$ power to detect a minimum difference of $16.6 \mathrm{~mm}$ in WOMAC 


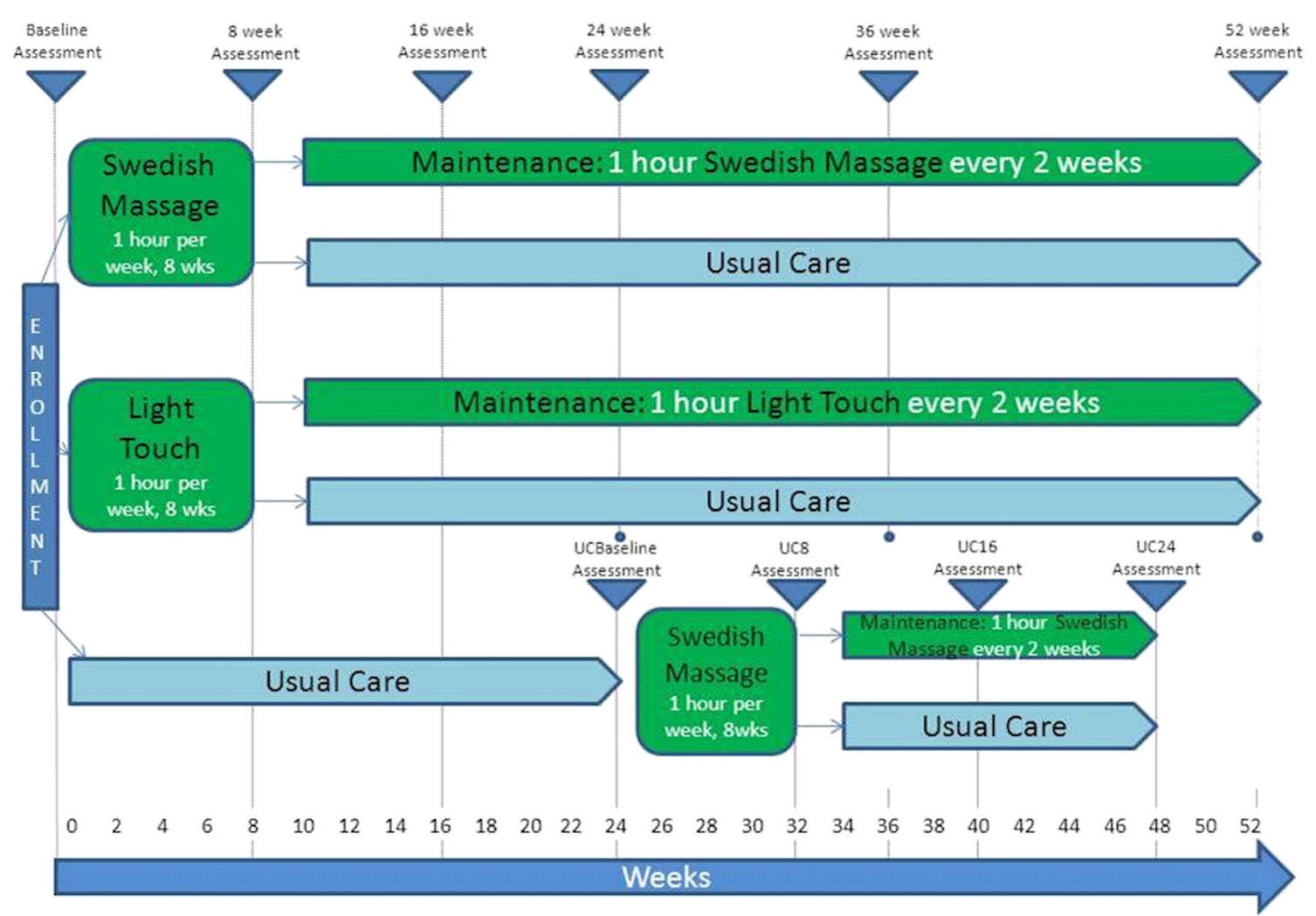

Figure 2 Schematic of study design.

between treatment groups, with maximum allowable type I error of 5\% (adjusted for 3 pairwise comparisons at 5 time points). ${ }^{19}$ A standard deviation of $21.0 \mathrm{~mm}$ in WOMAC and an attrition rate of $20 \%$ were used to compute the sample size.

\section{RESULTS}

Baseline characteristics of study participants are provided in Table 1. WOMAC data was available for $79 \%(n=175)$ of participants at the final assessment. Prescription medication and non-prescription pain medication usage were similar across groups (Supplemental Tables 3 and 4). Dropout rates differed by treatment arm. By the end of the study (week 52), 8 subjects dropped out who had originally been randomized to the massage arm (10.81\%), 25 from light-touch (34.25\%), and 7 from usual care followed by 8 -week massage ( $9.33 \%$ ). Many light-touch participants dropped out because they did not feel the intervention was beneficial, while others stated reasons unrelated to the intervention.

Table 1 Baseline Characteristics of Patient Sample, Overall and by Initial Randomization Group

\begin{tabular}{|c|c|c|c|c|}
\hline Characteristic & Swedish massage $(N=74)$ & Light-touch $(N=73)$ & Usual care $(N=75)$ & Total sample $(N=222)$ \\
\hline Age, year, mean $\pm \mathrm{SD}$ & $64.3 \pm 10.4$ & $62.8 \pm 10.4$ & $62.9 \pm 10.1$ & $63.3 \pm 10.3$ \\
\hline \multicolumn{5}{|l|}{ Sex, $n(\%)$} \\
\hline Female & $58(78.4)$ & $64(87.7)$ & $57(76.0)$ & $179(80.6)$ \\
\hline Male & 16 (21.6) & $9(12.3)$ & $18(24.0)$ & $43(19.4)$ \\
\hline \multicolumn{5}{|l|}{ Race, $n(\%)$} \\
\hline White & $62(83.8)$ & $64(87.7)$ & $64(85.3)$ & $190(85.6)$ \\
\hline Black or African American & $9(12.2)$ & $7(9.6)$ & $7(9.3)$ & $23(10.4)$ \\
\hline Asian & $3(4.1)$ & - & $4(5.3)$ & $7(3.2)$ \\
\hline Not reported & - & $2(2.7)$ & - & $2(0.9)$ \\
\hline \multicolumn{5}{|l|}{ Ethnicity, $n(\%)$} \\
\hline Hispanic or Latino & - & $1(1.4)$ & $1(1.3)$ & $2(0.9)$ \\
\hline Not Hispanic or Latino & $74(100)$ & $72(98.6)$ & $74(98.7)$ & $220(99.1)$ \\
\hline Body mass index, mean $\pm \mathrm{SD}$ & $30.4 \pm 6.9$ & $31.01 \pm 7.1$ & $30.8 \pm 7.6$ & $30.8 \pm 7.2$ \\
\hline \multicolumn{5}{|l|}{ Index knee, $n(\%)$} \\
\hline Right & $44(59.5)$ & $36(49.3)$ & $37(49.3)$ & $117(52.7)$ \\
\hline Left & $30(40.5)$ & $37(50.7)$ & $38(50.7)$ & $105(47.3)$ \\
\hline WOMAC, mean $\pm \mathrm{SD}$ & $48.8 \pm 18.6$ & $50.8 \pm 17.2$ & $53.9 \pm 16.8$ & $51.2 \pm 17.6$ \\
\hline
\end{tabular}

Values are mean $\pm S D$ except otherwise stated. All comparisons are non-significant $(p>0.05)$

$S D$, standard deviation; WOMAC, Western Ontario and McMaster Universities 


\section{Weeks}

From baseline to 8 weeks (Fig. 3, Table 2, Supplemental Fig. $1)$, the omnibus test of any group difference in the change in WOMAC Global was significant $(p=0.001, \mathrm{df}=2$ ). Massage significantly improved WOMAC Global scores compared to light-touch $(-8.16,95 \% \mathrm{CI}=-13.50$ to -2.81$)$ and usual care $(-9.55,95 \% \mathrm{CI}=-14.66$ to -4.45$)$. Additionally, massage improved pain, stiffness, and physical function WOMAC subscale scores compared to light-touch $(p<0.001 ; p=0.04$; $p=0.02$, respectively) and usual care $(p<0.001 ; p=0.002$; $p=0.002$; respectively). Omnibus tests of any group difference in change in WOMAC pain, stiffness, and function subscale scores were significant $(p<0.001, p=0.007, p=$ 0.004 , respectively; $\mathrm{df}=2$ ). Massage improved VAS ($11.20,95 \% \mathrm{CI}=-18.53$ to $-3.88, p=0.003)$, PROMIS-PI $T$-scores $(-2.09,95 \% \mathrm{CI}=-3.73$ to $-0.45, p=0.01)$, and 50 $\mathrm{ft}$ timed walk (time to walk $50 \mathrm{ft}$, in seconds; $0.16,95 \% \mathrm{CI}=$ 0.03 to $0.29, p=0.02$ ) compared to usual care, but not lighttouch. ROM (measured in degrees, using a goniometer) increased the most with massage, but was not significantly different compared to light-touch or usual care.

\section{Weeks}

Swedish Massage Versus Swedish Massage-Usual Care. At 52 weeks, the omnibus test of any group difference in the change in WOMAC Global from baseline to 52 weeks was not significant ( $p=0.707, \mathrm{df}=3$ ), indicating no significant difference in change between groups (Table 3). Omnibus tests of any group difference in change from baseline to 52 weeks were not significant for any of the other outcomes, so pairwise tests of significance are not reported. Additionally, massage maintenance did not significantly improve WOMAC Global, subscale scores, VAS, ROM, timed walk, and PROMIS-PI $T$ scores beyond the first 8 weeks, and compared to massageusual care at 52 weeks (massage change vs. massage-UC change $p$ value $=0.481)($ Supplemental Table 5).

Adverse Events. One hundred thirty-five total adverse events were reported; 133 (98.5\%) were rated mild or moderate, with 2 events rated severe. Only 1 severe event was determined to be likely resulting from the intervention (post-massage pain in subject's hip and thigh). Most commonly, mild and moderate adverse events were instances of knee pain following the massage or light-touch intervention and largely assessed as unlikely to be related to the intervention (e.g., common colds, bronchitis, minor car accidents, rashes, dental issues; Supplemental Table 6).

\section{DISCUSSION}

Our data indicate that 8 weeks of massage provided a statistically and clinically significant improvement of osteoarthritis symptoms. These results support findings from our earlier pilot study

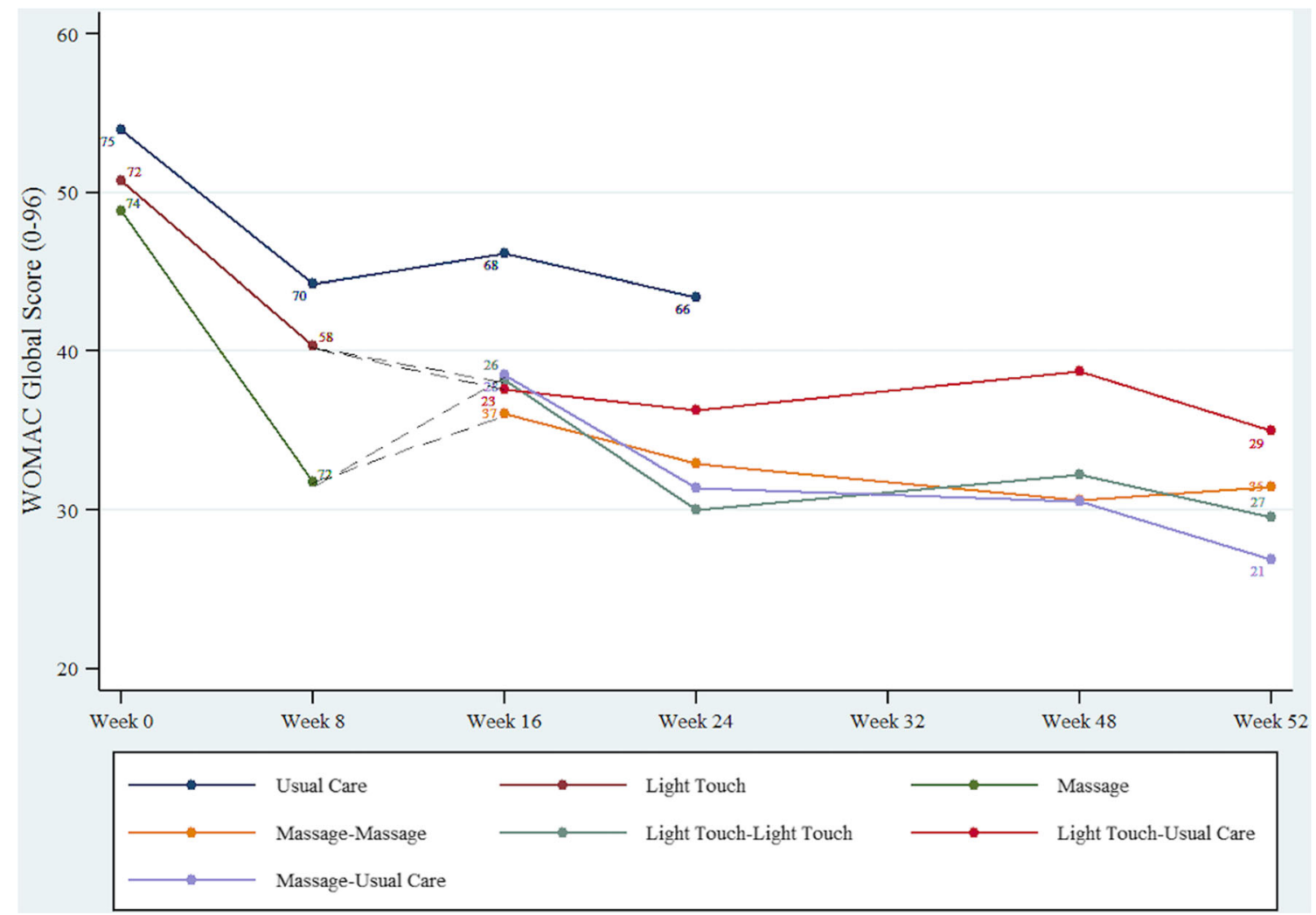

Figure 3 Unadjusted mean WOMAC Global scores. Higher scores on the WOMAC indicate worse outcome; N number of participants with primary outcome, WOMAC Global score. 
Table 2 Therapeutic Efficacy of Primary and Secondary Outcomes at 8 Weeks

\begin{tabular}{llll}
\hline \hline Outcome & \multicolumn{2}{l}{ Between-group improvement } & \\
\cline { 2 - 4 } & Swedish massage versus light-touch & Swedish massage versus usual care & Light-touch versus usual care \\
\hline WOMAC Global & $-8.16(-13.50$ to -2.81$)$ & $-9.55(-14.66$ to -4.45$)$ & $-1.40(-6.81 \text { to } 4.01)^{\mathrm{a}}$ \\
WOMAC pain subscale & $-10.98(-16.64$ to -5.31$)$ & $-10.83(-16.23$ to -5.43$)$ & $0.15(-5.57 \text { to } 5.86)^{\mathrm{a}}$ \\
WOMAC stiffness subscale & $-7.53(-14.54$ to -0.52$)$ & $-10.53(-17.23$ to -3.84$)$ & $-3.01(-10.07 \text { to } 4.06)^{\mathrm{a}}$ \\
WOMAC function subscale & $-6.24(-11.51$ to -0.97$)$ & $-8.15(-13.16$ to -3.14$)$ & $-1.91(-7.24 \text { to } 3.43)^{\mathrm{a}}$ \\
Visual analog scale & $-7.21(-14.93 \text { to } 0.52)^{\mathrm{a}}$ & $-11.20(-18.53$ to -3.88$)$ & $-4.00(-11.77 \text { to } 3.77)^{\mathrm{a}}$ \\
Range of motion $\left({ }^{\circ}\right)$ & $0.70(-2.33 \text { to } 3.74)^{\mathrm{a}}$ & $2.10(-0.78 \text { to } 4.98)^{\mathrm{a}}$ & $1.40(-1.66 \text { to } 4.45)^{\mathrm{a}}$ \\
Timed walk (ft/s) & $0.13(-0.01 \text { to } 0.27)^{\mathrm{a}}$ & $0.16(0.03$ to 0.29$)$ & $0.03(-0.11 \text { to } 0.17)^{\mathrm{a}}$ \\
PROMIS-PI T-score & $-0.79(-2.52 \text { to } 0.93)^{\mathrm{a}}$ & $-2.09(-3.73$ to -0.45$)$ & $-1.30(-3.04 \text { to } 0.45)^{\mathrm{a}}$ \\
\hline
\end{tabular}

The table displays mean and CI adjusted for baseline, study site, and BMI $(<30 / 30+)$. Higher scores on the WOMAC indicate worse outcome WOMAC, Western Ontario and McMaster Universities; PROMIS-PI, Patient-Reported Outcomes Measurement Information System-Pain Interference ${ }^{a} p>0.05$

that demonstrated safety, feasibility, and potential efficacy of 8 weeks of massage in reducing pain, stiffness, timed 50-ft walk, and improving physical function, compared to a wait-list control in adults with knee osteoarthritis. ${ }^{19}, 22$ We extend prior work by comparing massage to an active control and determining that a biweekly maintenance dose of massage maintained improvements in osteoarthritis symptoms at 52 weeks. Although mean group WOMAC for massage maintenance improved and massage followed by usual care got worse, this difference was not significant at 52 weeks with this sample size. Thus, while weekly massage provided more immediate clinically significant improvements in osteoarthritis symptoms at 8 weeks, massage and light-touch with or without biweekly maintenance showed similar improvements in WOMAC scores by the end of the study. The global WOMAC score has been validated for clinically significant change from baseline, but to our knowledge, has not been validated for clinical significance for between-group changes. ${ }^{30}$ Angst et al. validated a 7-point or $12 \%$ change from baseline on a 0 to 100 global WOMAC scale as clinically significant. ${ }^{30}$ If these criteria are applied to the between-group differences at 8 weeks in this study, massage compared to lighttouch (8.16 points and $17.2 \%$ difference) and massage compared to usual care (9.55 points and $20.9 \%$ difference), then the change in the massage group would be clinically significantly better than light-touch or usual care.

This study was designed with light-touch functioning as an active control for massage, providing relaxation, human touch, and interaction with a caring person, but no tissue manipulation. Our results suggest that light-touch may have significant therapeutic effects for individuals with painful knee osteoarthritis; however, reasons for this improvement are not well-understood. While the underlying mechanisms of massage-mediated improvements in osteoarthritis are not well-defined, preliminary studies suggest that massage may improve systemic immune and inflammatory profiles in healthy individuals. ${ }^{31}$ Future studies including comparison of mechanical, biochemical, and immunological effects of massage versus light-touch in people with osteoarthritis may help to elucidate mechanisms and optimize interventions.
Effect sizes were large in the present study and were comparable to, or superior to, those seen in other trials conducted using non-pharmacological approaches in patients with knee osteoarthritis. ${ }^{19,}$ 32-39 Massage does not carry the adverse effects of many pharmacologic and invasive treatments. ${ }^{6,7}$, $12,40,41$ While osteoarthritis treatment guidelines favor pharmacotherapy ${ }^{42}$ preferred treatments like NSAIDs and acetaminophen are frequently overused, increasing the risk of adverse effects such as gastrointestinal, cardiac, and renal complications. ${ }^{43,}{ }^{44}$ Such considerations argue for the use and study of non-pharmacological treatment approaches with advantageous safety and side effect profiles, promise of therapeutic efficacy, potential cost-effectiveness, and biological plausibility. Although the current analysis is limited in its ability to measure the long-term impact of massage on adverse medical events, the reduction in reported pain supports the potential that widespread adoption of this approach as a standard treatment could reduce reliance on pharmacotherapy.

\section{Limitations}

Study limitations include a relatively small and uniform sample. Despite conducting this study at geographically disparate sites, our sample was predominantly female and white. Although light-touch was described as a type of body work, it was difficult to blind our participants and massage therapists to the intended active intervention. Participant bias may have led to differential dropout rate and influenced estimates for the light-touch group, and it is possible that bias in the massage therapists could influence the administration of massage and light-touch. Between-group changes in WOMAC score have not been validated for clinical significance in this population, and criteria for evaluating these changes have varied; further investigation is warranted. Additionally, the study did not assess supplemental clinical data on study participants, such as comorbid conditions or psychological constructs that are common in patients with chronic pain (e.g., depression, anxiety, catastrophizing). These variables represent potentially important moderators of the treatment effect. 


\section{CONCLUSIONS}

This study demonstrated that a manualized protocol for a 60-min, whole-body Swedish massage, once weekly for 8 weeks, is safe and efficacious at reducing pain and increasing function in adults with knee osteoarthritis compared to an active control and usual care. Positive effects of 8 weeks of massage were quite durable without additional treatment. Long-term biweekly massage maintained gains from the initial 8 weekly treatments and had higher average global WOMAC score at all time points than without maintenance. However, due to significant improvements in all groups from baseline, massage maintenance was comparable to massage without maintenance and light-touch, with or without maintenance, at 52 weeks. Therefore, weekly massage can be used to facilitate more immediate improvement in symptoms of osteoarthritis, but maintenance biweekly massage did not provide significant additional benefit compared to no further therapy or to light-touch. This trial did not include a true usual care group beyond 24 weeks, so 8 weeks of weekly massage followed by biweekly maintenance compared to usual care alone at 52 weeks cannot be made. The safety and efficiency of symptom relief from 8 weeks of massage may allow individuals a more timely return to activities that enhance their quality of life and thus may be an attractive treatment option for many suffering from osteoarthritis of the knee.

\section{Acknowledgements:}

The team acknowledges the positive impacts and contributions of Ather Ali, ND, MPH, MHS, to their lives, with deep gratitude, love, and respect. We also thank Mary Carola (Rutgers), Michelle Pinto-Evans (Yale), and Gina Smith, MA (Yale), for coordinating study participants and entering data; Kim Turk, Kelly Cross, and Myra Blackwell (Duke); Lee Stang, Carol Nakagawara, Paula Jelly, and Susan Kmon (Yale); and Denise Ostopo-Gliozzi, Mariella Silva, and J. J. Long (Rutgers) for providing massage and light-touch interventions; Michael Patterson, $P h D, D O$, for developing the LT bodywork intervention; and the study subjects for their participation.

Funding Source: This study and publication were made possible by grant number R01ATO04623 from the National Center for Complementary and Integrative Health (NCCIH) at the National Institutes of Health.

Corresponding Author: Adam Perlman, MD, MPH; Duke Integrative Medicine, Durham, NC, USA (e-mail: adam.perlman@duke.edu).

\section{Compliance with Ethical Standards:}

All study materials were approved by the Institutional Review Boards of participating sites.

Conflict of Interest: The authors declare that they do not have a conflict of interest.

Disclaimer: Its contents are solely the responsibility of the authors and do not necessarily represent the official views of NCCIH.

Publisher's Note: Springer Nature remains neutral with regard to jurisdictional claims in published maps and institutional affiliations. 


\section{REFERENCES}

1. Lawrence RC, Felson DT, Helmick CG, et al. Estimates of the prevalence of arthritis and other rheumatic conditions in the United States. Part II. Arthritis Rheum. 2008;58(1):26-35.

2. Arden N, Nevitt M. Osteoarthritis:epidemiology. Best Pract Res Clin Rheumatol. 2006;20:3-25.

3. Prevalence of doctor-diagnosed arthritis and arthritis-attributable activity limitation - United States, 2010-2012. U.S. Department of Health and Human Services, Centers for Disease Control and Prevention; November 8, 2013.

4. Neogi T. The epidemiology and impact of pain in osteoarthritis. Osteoarthr Cartil. 2013;21(9):1145-1153.

5. Kato T, Xiang $\mathbf{Y}$, Nakamura $\mathbf{H}$, Nishioka $\mathbf{K}$. Neoantigens in osteoarthritic cartilage. Curr Opin Rheumatol. 2004; 16:604-608.

6. Fisher N, Pendergast D. Reduced muscle function in patients with osteoarthritis. Scand J Rehab Med. 1997;29:213-221.

7. Felson D. An update on the pathogenesis and epidemiology of osteoarthritis. Radiol Clin N Am. 2004;42:1-9.

8. Richmond $\mathbf{J}$, Hunter $\mathbf{D}$, Irrgang $\mathbf{J}$, et al. Treatment of osteoarthritis of the knee (nonarthroplasty). J Am Acad Orthop Surg. 2009;17(9):591600.

9. Hunter D, Felson D. Osteoarthritis. Br Med J. 2006;332:639-642

10. Matchaba P, Gitton $\mathbf{X}$, Krammer G, et al. Cardiovascular safety of lumiracoxib: A meta-analysis of all randomized controlled trials 1 week and up to 1 year in duration of patients with osteoarthritis and rheumatoid arthritis. Clin Ther 2005;27:1196-1214.

11. Naesdal J, Brown K. NSAID-associated adverse effects and acid control aids to prevent them: a review of current treatment options. Drug Saf 2006;29:119-132.

12. Messier S, Loeser R, Hoover J, Semble E, Wise C. Osteoarthritis of the knee: effects on gait, strength, and flexibility. Arch Phys Med Rehabil. 1992;73:29-36.

13. Ernst E. Complementary and alternative medicine for pain management in rheumatic disease Curr Opin Rheum 2002;14:58-62.

14. National Center for Complementary and Alternative Medicine. NCCAM backgrounder: massage therapy: an introduction. Vol NCCAM Publication No. D3272010.

15. Barnes PM, Bloom B, Nahin RL, Barnes PM, Bloom B, Nahin RL. Complementary and alternative medicine use among adults and children: United States, 2007. Natl Health Stat Rep. 2008(12):1-23.

16. Perlman A, Meng C. Rheumatology. In: Leskowitz E, ed. Complementary and alternative medicine in rehabilitation. St. Louis: Harcourt Heath Sciences; 2003:352-362.

17. Perlman A, Spierer M. Osteoarthritis. In: Rakel D, ed. Integrative medicine. Orlando: W.B. Saunders Co.; 2003:414-422.

18. Perlman A, Weisman R. Own your own health - the best of alternative and conventional medicine: pain Deerfield Beach: Health Communications, Inc.; 2006.

19. Perlman AI, Sabina A, Williams AL, Njike VY, Katz DL. Massage therapy for osteoarthritis of the knee: a randomized controlled trial. Arch Intern Med. 2006;166(22):2533-2538.

20. Clarke TC, Black LI, Stussman BJ, Barnes PM, Nahin RL. Trends in the use of complementary health approaches among adults: United States, 2002-2012. Natl Health Stat Rep. 2015(79):1-16.

21. Ali A, Kahn J, Rosenberger L, Perlman AI. Development of a manualized protocol of massage therapy for clinical trials in osteoarthritis. Trials. 2012;13:185

22. Perlman AI, Ali A, Njike VY, et al. Massage therapy for osteoarthritis of the knee: a randomized dose-finding trial. PloS One. 2012;7(2):e30248.

23. Altman R, Asch E, Bloch D, et al. Development of criteria for the classification and reporting of osteoarthritis: classification of osteoarthritis of the knee. Diagnostic and Therapeutic Criteria Committee of the American Rheumatism Association. Arthritis Rheum 1986;29(8):10391049.

24. Patterson M, Maurer S, Adler S, Avins A. A novel clinical-trial design for the study of massage therapy. Complement Ther Med. 2008;16(3):169176.
25. Bellamy N, Buchanan W, Goldsmith C, Campbell J, Stitt L. Validation study of WOMAC: a health status instrument for measuring clinically important patient relevant outcomes to antirheumatic drug therapy in patients with osteoarthritis of the hip or knee. J Rheumatol. 1988; 15:1833-1840.

26. Bellamy N. The WOMAC knee and hip osteoarthritis indices: development, validation, globalization and influence on the development of the AUSCAN hand osteoarthritis indices. Clin Exp Rheumatol. 2005;23(5 suppl 39):S148-S153.

27. McConnell S, Kolopack P, Davis A. The Western Ontario and McMaster Universities Osteoarthritis Index (WOMAC): a review of its utility and measurement properties. Arthritis Rheum. 2001;45(5):453-461.

28. Anagnostis C, Mayer TG, Gatchel RJ, Proctor TJ. The million visual analog scale: its utility for predicting tertiary rehabilitation outcomes. Spine. 2003;28(10):1051-1060.

29. Amtmann D, Cook KF, Jensen MP, et al. Development of a PROMIS item bank to measure pain interference. Pain. 2010;150(1):173-182.

30. Angst F, Aeschlimann A, Stucki G. Smallest detectable and minimal clinically important differences of rehabilitation intervention with their implications for required sample sizes using WOMAC and SF-36 quality of life measurement instruments in patients with osteoarthritis of the lower extremities. Arthritis Care Res 2001;45:384-391.

31. Rapaport MH, Schettler P, Bresee C. A preliminary study of the effects of repeated massage on hypothalamic-pituitary-adrenal and immune function in healthy individuals: a study of mechanisms of action and dosage. J Altern Complement Med. 2012;18(8):789-797.

32. Bhatia D, Bejarano T, Novo M. Current interventions in the management of knee osteoarthritis. J Pharm Bioallied Sci. 2013;5(1):30-38.

33. Anandacoomarasamy A, March L. Current evidence for osteoarthritis treatments. Ther Adv Musculoskelet Dis. 2010;2(1):17-28.

34. Wang $\mathbf{C}$, Schmid $\mathbf{C H}$, Hibberd PL, et al. Tai chi is effective in treating knee osteoarthritis: a randomized controlled trial. Arthritis Rheum. 2009;61(11):1545-1553.

35. Brismée JM, Paige RL, Chyu MC, et al. Group and home-based tai chi in elderly subjects with knee osteoarthritis: a randomized controlled trial. Clin Rehabil. 2007;21(2):99-111.

36. Tanaka R, Ozawa J, Kito N, Yamasaki T, Moriyama H. Evidence of improvement in various impairments by exercise interventions in patients with knee osteoarthritis: a systematic review and meta-analysis of randomized clinical trials. J Jpn Phys Ther Assoc. 2013;16(1):7-21.

37. Ni GX, Song $\mathbf{L}$, Yu B, Huang $\mathbf{C H}$, Lin JH. Tai chi improves physical function in older Chinese women with knee osteoarthritis. J Clin Rheumatol. 2010;16(2):64-67.

38. Tsai PF, Chang JY, Beck C, Kuo YF, Keefe FJ. A pilot clusterrandomized trial of a 20-week Tai Chi program in elders with cognitive impairment and osteoarthritic knee: effects on pain and other health outcomes. J Pain Symptom Manag. 2013;45(4):660-669.

39. Gignac G, Szodorai E. Effect size guidelines for individual differences researchers. Personal Individ Differ. 2016;102(November):74-78.

40. Kongtharvonskul J, Anothaisintawee T, McEvoy M, Attia J, Woratanarat $\mathbf{P}$, Thakkinstian $\mathbf{A}$. Efficacy and safety of glucosamine, diacerein, and NSAIDs in osteoarthritis knee: a systematic review and network meta-analysis. Eur J Med Res. 2015;20:24.

41. O'Neil CK, Hanlon JT, Marcum ZA. Adverse effects of analgesics commonly used by older adults with osteoarthritis: focus on non-opioid and opioid analgesics. Am J Geriatr Pharmacother 2012;10(6):331-342.

42. McAlindon TE, Bannuru RR, Sullivan MC, et al. OARSI guidelines for the non-surgical management of knee osteoarthritis. Osteoarthr Cartil. 2014;22(3):363-388.

43. Cryer B, Barnett MA, Wagner J, Wilcox CM. Overuse and misperceptions of nonsteroidal anti-inflammatory drugs in the United States. Am J Med Sci. 2016;352(5):472-480.

44. Szalavitz M. Study: taking just a little too much tylenol each time can be deadly. Pain. 2011. http://healthland.time.com/2011/11/23/study-taking-just-a-little-too-much-tylenol-each-time-can-be-deadly/. Accessed $11 / 5 / 18$ 